\title{
Relationships among social support, psychological and physical states among elderly Japanese women with breast cancer
}

\author{
Reiko Makabe ${ }^{1}$, Tohru Ohtake ${ }^{2}$, Tadashi Nomizu ${ }^{3}$ \\ 1. School of Nursing, Fukushima Medical University, Fukushima-shi, Fukushima, Japan. 2.School of Medicine, Fukushima \\ Medical University, Fukushima-shi, Fukushima, Japan. 3. Breast center, Hoshi General Hospital, Koriyama-shi, Fukushi- \\ ma, Japan
}

Correspondence: Reiko Makabe, RN, PhD, School of Nursing, Fukushima Medical University, Hikarigaoka, Fukushimashi, Fukushima, Japan 960-1295. Email: makabe@fmu.ac.jp.

Received: Sept 14, 2011

DOI : $10.5430 /$ jnep.v1n1p4

\begin{abstract}
Accepted: October 8, $2011 \quad$ Published: December 1, 2011
\end{abstract}
URL: http://dx.doi.org/10.5430/jnep.v1n1p4

\section{Abstract}

Background/Objective: Although some studies conducted in Western cultures have identified positive influences of social support on the psychological and physical states of elderly women with breast cancer, few studies have investigated the relationships between social support and its effects on health outcomes in elderly Japanese women with breast cancer. The purpose of this study was to describe and investigate the relationships among social support, social support network, and psychological and physical states among elderly Japanese women with breast cancer.

Methods: Elderly Japanese women with breast cancer $(n=65)$ participated in the study. Measures used in this study were the Japanese versions of instruments with established reliability and validity: (1) the Social Support Scale for Japanese Elderly (SSSJE); (2) the Visual Analogue Scale for Social Support (VASSS); (3) the General Health Questionnaire (GHQ) and (4) the Physical States Interview Form (PSIF). Data analysis included descriptive, $t$-tests, and Pearson's correlations.

Results: Descriptive statistics showed that the size of the social support network ranged from 2 to 34 (M=9.09; SD=6.57). Comparisons of psychological states showed a significant difference between the women who were over and under 70 years old. Psychological states significantly correlated with social support network and conflict among elderly Japanese women with breast cancer.

Conclusions: The results reveal that there are significant relationships among social support, social support network, and psychological and physical states among elderly Japanese women with breast cancer. The results reveal that there are significant relationships among social support, social support network, and psychological and physical states among elderly Japanese women with breast cancer. Nurses should provide information on social support as an important factor to help elderly Japanese women with breast cancer.

\section{Key words}

Social support, Psychological states, Physical states, Elderly Japanese women, Breast cancer 


\section{I ntroduction}

Social support has been recognized as an important component of psychological and physical health [1]. It is known to not only directly affect stress and health outcomes, but also buffer the effects of stress on health outcomes among cancer patients. Studies of social support [2-4] and social support network [5] have reported the positive effects of social support on health outcomes among women with breast cancer. Also, longitudinal studies of social support for breast cancer patients found significant relationships between social support and psychological states [6]. In addition, intervention studies of social support for breast cancer patients have reported significant positive influences of support intervention programs on patients' psychological states [7, 8]. There have been qualitative, descriptive and comparative, and correlational studies on elderly women with breast cancer in Western countries. Tilden, Hirsch, and Nelson (1994) stated that studies of social support should include both its positive and negative aspects [9].

Cancer has been the leading cause of death in Japan since 1981, and the death rate specifically due to breast cancer is increasing [10]. There are increasing incidences of breast cancer, and it is the most common cancer diagnosed among Japanese women [11].

Despite this, few studies have investigated the relationships between social support and its effects on health outcomes in Japanese women [12, 13]. Social support requires interpersonal relationships, which are influenced by beliefs, values, and behavior [14]. Studies of social support among breast cancer patients conducted in other countries indicate that the experience of breast cancer differs among cultures [15-17]. Some studies conducted in Western cultures have identified positive influences of social support on the psychological and physical states of elderly women with breast cancer $[3,18]$.

Because unique cultural characteristics are shown to be an important factor, the results of social support studies conducted in other cultures should be considered with caution for Japanese populations. A qualitative study of social support among Japanese women with breast cancer described reciprocity. It is related to "giri," a Japanese term referring to social obligation and to valuing the maintenance of harmonious relationships, as one of the unique components of social support in this population [19]. In addition, age was expressed as another factor among the Japanese population, the interpersonal relationships change with life stages, and social support networks in elderly Japanese populations mainly include family members [20]. Thus, a study of social support, social support networks, and health outcomes (i.e., psychological and physical states) among elderly Japanese women with breast cancer and that considers the cultural issues of social support is necessary to provide a basis for assessment and intervention.

\section{Purpose}

The purpose of this study was to describe and investigate the relationships among social support, social support network, and psychological and physical states among elderly Japanese women with breast cancer.

\section{Conceptual framework}

The conceptual framework used in this study was based on House [1], focusing on social support effects on psychological and physical states among elderly Japanese women with breast cancer (Figure 1). Specifically, social support was perceived as having four components: 1) emotional support was defined as the perceived availability or enactment of helping behaviors by members of the social network; 2) instrumental support was defined as the perceived availability of tangible goods or services; 3) reciprocity was defined as the perceived availability or occurrence of an exchange of emotional or tangible goods or services, or the returning of emotional or tangible goods or services; 4) conflict was defined as perceived discord or stress in relationships caused by behaviors of others or by the absence of behaviors of others, such as withholding help [9]. Moreover, a social support network was defined as the sources of social support provided by others, such as significant persons in an individual's life. 


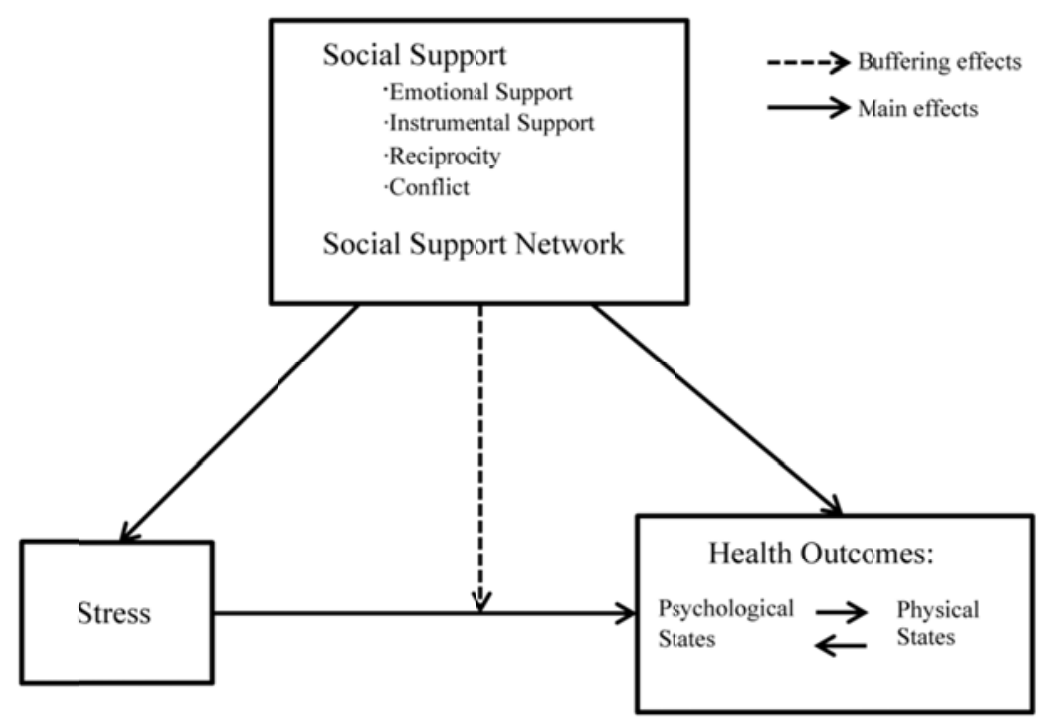

Figure 1. The Conceptual Framework: The Relationship of Stress, Social Support, Social Support Network and Health Outcomes: Psychological and Physical States

Health outcomes were viewed as both psychological and physical states. Derived from Lazarus and Folkman [21], the term psychological states was defined as an individual's psychological symptoms being the result of the relationships between a person and the environment that are appraised by the person as taxing or exceeding his or her resources and endangering his or her well-being. Physical states were defined as the physical signs and symptoms commonly experienced by women who have had treatment for malignant breast disease [22, 23].

\section{Methods}

\subsection{Research design}

This descriptive study was designed to describe and investigate the relationships of major variables of social support among elderly Japanese women with breast cancer.

\subsection{Subjects and setting}

A convenience sample of elderly Japanese female breast cancer patients, who were receiving treatment for breast cancer at general hospitals located in northern Japan, participated in the study. The subjects included elderly Japanese women who were older than 65, who were born and had lived their entire lives in Japan, who had been diagnosed with breast cancer and had undergone breast surgery, and who followed in an outpatient setting. The age of 65 and over was decided to be old age since it has been used in Japan [10]. Potential subjects who were diagnosed with psychiatric disorders were excluded from this study.

\subsection{Measurements}

To measure social support, social support network, psychological states, and physical states, the Japanese versions of four instruments with established reliability and validity were used: (1) the Social Support Scale for Japanese Elderly (SSSJE); (2) the Visual Analogue Scale for Social Support (VASSS); (3) the General Health Questionnaire (GHQ) and (4) the Physical States Interview Form (PSIF). Subjects also reported their own personal and illness-related data. 


\subsubsection{Social support and social support network}

The SSSJE, was used to measure social support, which was established reliability (i.e., Cronbach's alphas) and validity (i.e., constructed validity) by a factor analysis approach [24]. The SSSJE was designed to measure emotional support, instrumental support, reciprocity, and conflict. In this study, the subscales of emotional support, instrumental support, and reciprocity were used. The subscale of conflict was not used because of an inadequate alpha in the premier analysis of this study. For this study, the reliabilities for the scale and subscales, i.e., alphas, ranged from 0.768 to 0.825 . The VASSS was used to measure conflict. Also, the subjects' social support network was obtained by listing people whom the subjects considered significant others. In addition, the data of contacts with other breast cancer patients, which meant maintaining ongoing contacts with other breast cancer patients, personally and individually, were obtained by "Yes" or "No" responses.

\subsubsection{Psychological states}

The GHQ was used to measure the psychological states of the subjects. The GHQ was developed by Goldberg and others, who established the instrument's reliability and validity [25, 26]. Nakagawa and Daibou [27] translated the GHQ from English into Japanese (Japanese GHQ (JGHQ)), and they reported its established reliability alpha and construct and concurrent validities. The GHQ has five versions: the 60-, 30-, 28-, 20-, and 12-item GHQs. The total score of the 28-item JGHQ was used because it is a shorter version, and is widely used as an indication of psychological distress, disturbance, states, and health. The range of the total score in this method was 0 to 28, and a low score indicated a better psychological state. In this study, the alpha was 0.718 .

\subsubsection{Physical states}

The PSIF was developed with an expert oncology nursing researcher to obtain information about the physical signs and symptoms commonly experienced by women who have had treatments for malignant breast disease [19, 23]. The subjects responded "Yes" or "No" to each item and described the signs and symptoms if they answered "Yes." After performing content analysis of their descriptions of signs and symptoms, a cumulative score of "Yes" responses was used to indicate the patients' physical state. Possible scores ranged from 0 to 10, with a low score indicating a better physical state. To establish the intra-rater reliability of the scale, the primary investigator performed the content analysis, categorizing of raw data, twice with a two-week interval, and the results of the content analysis were the same on both occasions.

\subsection{Data collection procedures}

Prior to the initiation of the study, Fukushima Medical University and hospital research ethical committees reviewed and approved the study to ensure the protection of human subjects. Elderly Japanese women with breast cancer were recruited from hospitals in northern Japan. Data were collected once at out-patient settings. During the data collection period, the primary investigator explained to the potential subjects who met the inclusion criteria, the purpose of the study, procedures, risks and benefits, confidential treatment of any information received, the voluntary nature of participation, and the lack of any penalty for withdrawal from the study. Before giving consent, potential subjects had an opportunity to ask questions about the study and to refuse to participate. After the consent was given, they completed the study instruments.

\subsection{Data analysis}

Data analysis included descriptive and inferential statistics, e.g., $t$ - tests and Pearson's correlations. Descriptive statistics were used to describe the subjects and mean scores of the major variables. As inferential statistics, $t$ - tests were used for comparisons with the social support, social support network, psychological state, and physical state variables between age (65 to 70 years old vs. over 71 years old) and contacts with other breast cancer patients (Yes or No). The ages of 70 and 71 were selected as a cut-off to divide into two groups as young old and others because of the mean age of 71.9 in this study. Pearson's correlations were calculated to explore correlations among the major variables. Data were analyzed using the statistical software package SPSS18.0 (SPSS Inc.). Significance was set at $p<0.05$. 


\section{Results}

\subsection{Characteristics of the subjects}

Sixty-five women consented to participate in the study and completed the instruments. Table 1-1 shows their demographic data. The mean age of the sample was 71.9 years $(S D=5.5$, range $=20$, from 65 to 85). Most of the subjects were married, and had been employed in the past. At the time of data collection, fifteen subjects (23.1\%) were self-employed, full-time employed, and part-time employed. About half of them $(n=30,46.2 \%)$ had contacts with other breast cancer patients.

Table 1-2 summarizes their illness-related data. The subjects received breast surgery as treatment for breast cancer including lumpectomy, modified radical mastectomy, or mastectomy. The mean number of months after breast surgery was 45.0 months. Most were classified as stage I or stage II, in the early stage of the disease. They received postoperative adjuvant treatments of chemotherapy, radiation therapy, and/or hormonal therapy. About two thirds of them had other medical problems, such as arthritis, hypertension, diabetes and/or other non-cancer health problems.

The total score of signs and symptoms related to breast cancer and breast treatment ranged from 0 to 4 . About one-third of subjects had no signs or symptoms, and the others had one, two, three signs or symptoms. Examples of signs and symptoms were fatigue, pain in the affected side of the breast, abnormal breast sensations, arm sensations, edema, and other symptoms. Social support network comprised mainly family members (i.e., spouses, daughters, sons, sisters, brothers, grandchildren, and in-laws), friends, neighbors, other breast cancer patients, health care professionals, and others. The mean social support network size was 9.09. Among the married participants, 42 women listed their spouses as a part of their social support network. Two participants (3.1\%) listed health care professionals, i.e., a surgeon; however, no participant listed a nurse as part of their social support network.

The objectives of the study were to describe and investigate the relationships among social support, social support network, psychological and physical states among elderly Japanese women with breast cancer. Specific research questions were the following: 1) what were differences in the main variables? and 2) what were correlations among the main variables?

Table 1-1. Characteristics of the Subjects: Personal Data $(n=65)$

\begin{tabular}{|c|c|c|c|}
\hline Item & Detail & & \\
\hline \multirow{5}{*}{$\begin{array}{l}\text { Age } \\
{[\mathrm{M}=71.9 ;} \\
\mathrm{SD}=5.5 ; \\
\text { Range = } 20 \text { (65-85)] }\end{array}$} & Range & $\mathrm{n}$ & $\%$ \\
\hline & $65-69$ & 24 & 36.9 \\
\hline & $70-74$ & 23 & 35.4 \\
\hline & 75-79 & 10 & 15.4 \\
\hline & 80-85 & 8 & 12.3 \\
\hline \multirow{4}{*}{$\begin{array}{l}\text { Marital Status } \\
\text { [Married subjects' (n=49) } \\
\text { marital years M = 45.0; } \\
\mathrm{SD}=7.00 ; \\
\text { Range = } 39(23-62)]\end{array}$} & Status & $\mathrm{n}$ & $\%$ \\
\hline & Never Married & 1 & 1.5 \\
\hline & Married & 49 & 75.4 \\
\hline & Widowed & 15 & 23.1 \\
\hline
\end{tabular}


Table 1-1. (Continued.)

\begin{tabular}{|c|c|c|c|}
\hline Item & Detail & & \\
\hline \multirow{7}{*}{ Education } & Education & $\mathrm{n}$ & $\%$ \\
\hline & Junior high school & 20 & 30.8 \\
\hline & High school & 25 & 38.5 \\
\hline & Junior college & 2 & 3.1 \\
\hline & Technical school & 7 & 10.8 \\
\hline & ED under the Old System & 9 & 13.8 \\
\hline & Missing & 2 & 3.1 \\
\hline \multirow{3}{*}{ Employment Status: Past } & Status & $\mathrm{n}$ & $\%$ \\
\hline & No & 4 & 6.2 \\
\hline & Yes & 61 & 93.8 \\
\hline \multirow{3}{*}{ Employment Status: Present } & Status & $\mathrm{n}$ & $\%$ \\
\hline & No & 50 & 76.9 \\
\hline & Yes & 15 & 23.1 \\
\hline \multirow{3}{*}{$\begin{array}{l}\text { Contact with other Breast } \\
\text { Cancer Patients }\end{array}$} & Status & $\mathrm{n}$ & $\%$ \\
\hline & Yes & 30 & 46.2 \\
\hline & No & 35 & 53.8 \\
\hline
\end{tabular}

Note: Because of rounding, percentages may not total 100.

Table 1-2. Characteristics of the Subjects: Illness-Related Data $(\mathrm{n}=65)$

\begin{tabular}{llll}
\hline Item & Detail & & \\
\hline & Stages & $\mathrm{n}$ & $\%$ \\
Stages of Breast Cancer & 0 & 2 & 3.1 \\
& I & 31 & 47.7 \\
& II & 28 & 43.1 \\
& Missing & 4 & 6.2 \\
Types of Breast Surgery & Type & $\mathrm{n}$ & $\%$ \\
& Lumpectomy & 37 & 56.9 \\
& Modified Radical Mastectomy & 27 & 41.5 \\
& Mastectomy & 1 & 1.5 \\
\hline
\end{tabular}

(Table 1-2 continued on page 10.) 
Table 1-2. (Continued.)

\begin{tabular}{|c|c|c|c|}
\hline Item & Detail & & \\
\hline \multirow{7}{*}{$\begin{array}{l}\text { Months after Breasts Surgery } \\
{[\mathrm{M}=45.0 ;} \\
\text { SD = 55.7; } \\
\text { Range = } 323(1-324)\end{array}$} & Months & $\mathrm{n}$ & $\%$ \\
\hline & -12 & 18 & 27.7 \\
\hline & $13-24$ & 11 & 16.9 \\
\hline & $25-36$ & 9 & 13.8 \\
\hline & $37-48$ & 6 & 9.2 \\
\hline & $49-60$ & 8 & 12.3 \\
\hline & $61-$ & 13 & 20.0 \\
\hline \multirow{3}{*}{$\begin{array}{l}\text { Chemotherapy } \\
\text { (Adjuvant therapy) }\end{array}$} & State & $\mathrm{n}$ & $\%$ \\
\hline & Yes & 11 & 16.9 \\
\hline & No & 54 & 83.1 \\
\hline \multirow{3}{*}{$\begin{array}{l}\text { Radiotherapy } \\
\text { (Adjuvant therapy) }\end{array}$} & State & $\mathrm{n}$ & $\%$ \\
\hline & Yes & 11 & 16.9 \\
\hline & No & 54 & 83.1 \\
\hline \multirow{3}{*}{ Hormone therapy } & State & $\mathrm{n}$ & $\%$ \\
\hline & Yes & 42 & 64.6 \\
\hline & No & 23 & 35.4 \\
\hline \multirow[t]{3}{*}{ Other Medical Problems } & State & $\mathrm{n}$ & $\%$ \\
\hline & Yes & 47 & 72.3 \\
\hline & No & 18 & 27.7 \\
\hline
\end{tabular}

Note: Because of rounding, percentages may not total 100.

\subsection{Comparisons}

Comparisons of main variables showed significant differences in conflict between the subjects who contacted women with breast cancer and the subjects who did not. Conflict the group of women who contacted other breast cancer patients was higher than in the group of women who did not contact other breast cancer patients. Also, the variable of physical states was significantly different between the two groups of subjects who contacted other breast cancer patients and those who did not. In addition, psychological states were significantly higher for the women who were aged 71 and above than for those aged 70 and below (Table 2). 
Table 2. Comparisons of Main Variables: Conflict, Physical States, and Psychological States ( $\mathrm{n}=65)$

\begin{tabular}{lllll}
\hline Dependent & Independent & $\mathbf{n}$ & $\mathbf{M}(\mathbf{S D})$ & $\boldsymbol{t}(\mathbf{d f})$ \\
\hline \multirow{2}{*}{ Conflict } & No contact with other pts & 32 & $16.16(18.14)$ & $-2.00(58)^{*}$ \\
& Contact with other pts & 30 & $29.50(32.33)$ & \\
Physical States & No contact with other pts & 35 & $1.06(1.05)$ & $-2.29(63) *$ \\
& Contact with other pts & 30 & $1.70(1.21)$ & \\
Psychological States & Under 70 years old & 24 & $1.88(1.65)$ & $2.12(61)^{*}$ \\
& Over 71 years old & 39 & $3.33(3.10)$ & \\
\hline
\end{tabular}

Notes: Significance was set at $* P<0.05$

\subsection{Correlations}

Table 3 shows the results of Pearson's correlations among the main variables. Age and social support network correlated significantly. Psychological states were significantly correlated with social support network and conflict. However, physical states and psychological states were not significantly correlated (Table 3).

Table 3. Pearson's Correlations of Main Variables ( $\mathrm{n}=65)$

\begin{tabular}{llllllllll}
\hline Items & $\mathbf{1}$ & $\mathbf{2}$ & $\mathbf{3}$ & $\mathbf{4}$ & $\mathbf{5}$ & $\mathbf{6}$ & $\mathbf{7}$ & $\mathbf{8}$ & $\mathbf{9}$ \\
\hline Age & --- & & & & & & & & \\
Contact with Other pts & -.09 & --- & & & & & & & \\
Social Support Network & $-.27^{*}$ & .09 & --- & & & & & & \\
Emotional Support & -.22 & $.28^{*}$ & .17 & --- & & & & & \\
Instrumental Support & -.24 & $.45^{* *}$ & .05 & $.51^{* * *}$ & --- & & & & \\
Reciprocity & .04 & .10 & .03 & -.13 & .03 & --- & & & \\
Conflict & -.23 & $.25^{*}$ & .01 & -.20 & -.12 & .09 & --- & & \\
Physical States & -.14 & $.28^{*}$ & -.04 & .10 & -.05 & .20 & .25 & --- & \\
Psychological States & .19 & -.02 & $-.28^{*}$ & -.24 & -.17 & -.10 & $.28^{*}$ & .20 & --- \\
\hline Notes: All significant levels were based on a two-tailed test. ${ }^{*} P<0.05, * * P<0.011^{* * * *}<0.001$ & & & & & &
\end{tabular}

\section{Discussion}

This study was conducted to describe and investigate relationships among social support, social support network, and psychological and physical states among elderly Japanese women with breast cancer. The results revealed not only the existence of significant differences, but also significant correlations, in factors used in the study sample, as discussed below.

\subsection{Social support and social support network}

Makabe and Nomizu (2007) conducted a social support longitudinal study of Japanese women with breast cancer who were receiving follow-up medical care at outpatient settings [22]. They reported that the average social support network Published by Sciedu Press 
size was 11.3, while that in the present study was 9.09, during the first year after being diagnosed with breast cancer. The mean age of the subjects in this study was 53.11 years, while that in the present study was 71.9 . Thus, comparing the two social support network sizes between the two studies, the older population was smaller. Also, as in this study, previous studies found a significant negative correlation between age and social support network. The research results were consistent with the social support network size and age.

The findings regarding social support networks in the present study are consistent with those of Kobayashi (2008) [28] and Hashimoto (2005) [20]. Kobayashi (2008) studied some characteristics of social support networks among elderly Japanese, and she reported their social support networks mainly included family members who lived in the same household [28]. Hashimoto (2005) noted that interpersonal relationships change with life stage and that in elderly Japanese populations, the social support network mainly comprised family members [20]. Moreover, the subjects' social support network and psychological states showed a significant negative correlation. The results from this study revealed that a stronger social support network was related to lower psychological state scores, and better psychological states. Parrish and others (2005) found a similar result that having more friends and relatives for support was significantly related to lower levels of depression. The results suggest that social support is important for their psychological states [29].

Most of our study subjects were married women, and most listed their spouses as social support network members. Northouse (1989) found that support from family members, especially from spouses, was the most important factor that changed their breast cancer experience [6]. Stenberg and others (2009) reported in their literature review on the effects of caring for patients with cancer. They reported that family caregivers experience many difficult problems and increased responsibilities during and after the patient undergoes treatment and rehabilitation for cancer [30]. Thus, future research studies are needed to clarify social support and social support networks both elderly Japanese women with breast cancer and their family members.

Experiencing the disease and receiving treatment became a social activity. In this study, the participants, in their social support network, listed their health care professionals, as well as other breast cancer patients. However, nurses were not listed in their social support network. Different from this study's results, Goodwin and others (2003) reported that women with indicators of poor social support were more likely to benefit from nurse case management [31]. These different results need to be clarified in a future study, including the social and cultural issues related to delivery of medical services in Japan.

\subsection{Physical and psychological states}

As for physical states, the total score of signs and symptoms related to breast cancer and treatment for malignant breast disease ranged from 0 to 4 . One-third of the subjects had no signs or symptoms, while two-third of the sample had 1 to 4 signs and symptoms, such as fatigue, pain in the affected side of the breast, abnormal breast sensations, arm sensations, and edema. Von Ah, Kang, and Carpenter (2008) reported that fatigue was the most common symptom among women with breast cancer, consistent with the reports [32].

Pearson's correlations of physical states with contacts with other breast cancer patients were significant. Having more signs and symptoms was significantly correlated with contacts with other breast cancer patients. However, physical and psychological states were not significantly correlated. Makabe and Nomizu (2007) conducted a longitudinal study to investigate the relationships among social support and psychological and physical states in Japanese women with breast cancer aged between 30 and 90 years (mean age, 53.11). They reported a significant correlation between physical and psychological states. This was not consistent with the study of Makabe and Nomizu (2007) [22]. Therefore, this issue needs to be clarified in further studies.

Also, the variable of physical state was significantly different between the two groups of subjects who had contacts with other breast cancer patients and those who did not. A higher score for physical states was found for those who had contacts 
with other breast cancer patients than those without such contacts. In addition, contacts with other breast cancer patients were significantly correlated with not only subscales of social support such as emotional, instrumental, and conflict, but also physical states. These results were consistent with a previous longitudinal study of Japanese women aged between 30 and 90 years with breast cancer who had contact with other breast cancer patients (Makabe and Nomizu 2007) [22]. In the future, the relationships among population factors should be investigated by conducting longitudinal studies.

Comparisons of main variables showed significant differences. Women who were over 71 years old showed significant differences in psychological states compared with those who were under 70 years old. Psychological state scores were significantly higher in the women who were over 71 years old than those under 70 . Those under 70 years old had lower psychological state scores, and thus better psychological states.

\subsection{Conflict}

Contacts with other breast cancer patients showed in higher score in conflict. Also, conflict was significantly correlated with psychological states. A lower score for conflict was significantly correlated with a lower score for psychological states, and thus, better psychological states. In a qualitative study, Makabe and others (2010) found elderly Japanese women with breast cancer had conflict experiences prior to their own hospitalization to receive treatment for cancer [33]. They were caring for family members with health problems, e.g., cancer, heart disease, and cognitive disorders. The results were consistent with other studies among Japanese women with breast cancer, although these studies focused on subjects aged between 30 and 90 [22, 31]. Therefore, the results indicate that nurses need to assess not only their patients, but also patients' family members, which shows the need for further studies of these aspects.

\section{Summary}

This study provides an understanding of the relationships among social support, social support network, and psychological and physical states among elderly Japanese women with breast cancer. The conclusions are the followings:

1) The size of the social support network ranged from 2 to 34, and this was smaller than that in previous studies that focused on Japanese women with breast cancer. The results revealed that social support from family members was important for psychological states of elderly Japanese women with breast cancer.

2) Comparisons of psychological states related to demographic variables were significantly different between the women who were under 70 years old and those over 71 years old. The correlation between age and social support network size were significantly negative.

3) Psychological states were significantly correlated with social support network and conflict, which implies that better psychological states were associated with larger social support networks. A lower score for conflict was correlated with better psychological states. Having signs and symptoms was associated with having more contacts with other breast cancer patients. Physical states correlated significantly with contacts with other breast cancer patients.

4) Therefore, nurses need to assess not only elderly Japanese women with breast cancer, but also patients' family members, because they were important for providing social support as a social support network.

\section{Limitations}

There were some limitations to this study. First, our respondents were largely homogenous in terms of marital status, and most subjects were mainly in the early stage of the disease, so these findings are not applicable to elderly women who live alone, or to stages III or IV patients. Second, the length of time after breast surgery was 1 to 324 months, so the study results should be carefully interpreted. Third, because this study was a cross-sectional study, the results identified only 
comparisons and/or correlations of the main variables. Also, the sample size was 65 , so interpretation of the results requires caution.

\section{Clinical implications and future nursing research}

Findings from this study indicate to a need for additional research to investigate the relationships among social support, social support network, and psychological and physical states among elderly Japanese women with breast cancer. Nurses should provide information on social support, which is as an important factor to help elderly Japanese women with breast cancer.

Finfgeld-Connett (2005) stated that nurses should encourage patients to use and enhance personal support networks to increase social support results and improve mental health [34]. The results of this study suggest that nurses should assess the levels of different types of social support and their relationship with patients' psychological and physical states, thereby improving women's health outcomes. Considering cultural differences, nurses assessing patients and their family members, and having contacts with a woman's family members, may facilitate their adjustment and prepare them for a support role.

In the future, considering additional cultural characteristics, studies of social support should focus on social support, social support networks, psychological and physical states, and especially different population demographics, including marital status and stage of breast cancer. Also, future studies need to consider not only elderly Japanese women with breast cancer, but also their family members. Prospective longitudinal studies are needed to investigate correlations and comparisons of social support, social support network, psychological and physical states among elderly Japanese women with breast cancer.

\section{Acknowledgement}

We would like to thank the subjects who participated in the study.

\section{Research funding}

The research was funded by Japan Society for the Promotion of Science: Grant-in-Aid for Scientific Research (C): \# MO19592503.

\section{References}

[1] House JS. Work stress and social support. Menlo Park, CA: Addison-Wesley, 1981.

[2] Han WT, Collie K, Koopman C, Azarow J, Classen C, Morrow GR et al. Breast cancer and problems with medical interactions: Relationships with traumatic stress, emotional self-efficacy, and social support. Psycho-Oncol. 2005; 14: 318-330. PMid:15386762 http://dx.doi.org/10.1002/pon.852

[3] Maly RC, Umezawa Y, Leake B, Silliman RA. Mental health outcomes in older Women with breast cancer: Impact of perceived family support and adjustment. Psycho-Oncol. 2005; 14:535-545. PMid: 15493064 http://dx.doi.org/10.1002/pon.869

[4] Tan M, Karabulutlu E. Social support and helplessness in Turkish with cancer. Cancer Nurs. 2005; 28:236-240. PMid:15915069 http://dx.doi.org/10.1097/00002820-200505000-00013

[5] Kroenke CH, Kubzansky LD, Schernhammer ES, Holmes MD, Kawachi I. Social networks, social support, and survival after breast cancer diagnosis. J of Clinical Oncol. 2006; 24:1105-1111. PMid:16505430 http://dx.doi.org/10.1200/JCO.2005.04.2846

[6] Northouse L. A longitudinal study of the adjustment of patients and husbands to breast cancer. Oncol Nurs Forum. 1989; 16:511-516. PMid:2755858

[7] Coleman E A, Tulman L, Samarel N, Wilmoth MC, Rickel L, Rickel M, et al. The effect of telephone social support and education on adaptation to breast cancer during the year following diagnosis. Oncol Nurs Forum. 2005; 32:822-829.

http://dx.doi.org/10.1188/05.ONF.822-829 
[8] Northouse L, Kershaw T, Mood D, Schafenacker A. Effects of a family intervention on the quality of life of women with recurrent breast cancer and their family caregivers. Psycho-Oncol. 2005; 14:478-491. PMid: 15599947 http://dx.doi.org/10.1002/pon.871

[9] Tilden VP, Hirsch AM, Nelson CA. The Interpersonal Relationship Inventory: continued psychometric evaluation. J of Nurs Measurement. 1994; 2:63-78. PMid:7882093

[10] Health and Welfare Statistics Association.Trends of age-specific incidence rates per 100,000 populations in Japan according to sex, primary sites, and year. Journal of Health and Welfare Statistics. 2008; 55:410-411.

[11] Fukutomi T. Nyusenngeka [Breast surgery]. Gendai Igaku. 2008; 56:29-32.

[12] Makabe R, Nomizu T. Social support and psychological and physical states among Japanese patients with breast cancer and their spouses prior to surgery. Oncol Nurs Forum. 2006; 33:651-655. PMid:16676022 http://dx.doi.org/10.1188/06.ONF.651-655

[13] Miyashita M. A randomized intervention study for breast cancer survivors in Japan. Cancer Nurs. 2005; 28:70-78. PMid: 15681985 http://dx.doi.org/10.1097/00002820-200501000-00011

[14] Hamilton JB, Sandelowski M. Types of social support in African Americans with cancer. Oncol Nurs Forum. 2004; 31:792-800. PMid: 15252433 http://dx.doi.org/10.1188/04.ONF.792-800

[15] Ashing-Giwa KT, Padilla G, Tejero J, Kraemer J, Wright K, Coscarelli A, et al. Understanding the breast cancer experience of women: a qualitative study of African American, Asian American, Latina and Caucasian cancer survivors. Psycho- Oncol. 2004; 13:408-428. PMid:15188447 http://dx.doi.org/10.1002/pon.750

[16] Davis C, Williams P, Parle M, Redman S. Assessing the support needs of women with early breast cancer in Australia. Cancer Nurs. 2004; 27:169-174. PMid: 15253175 http://dx.doi.org/10.1097/00002820-200403000-00011

[17] Kagawa-Singer M, Wellisch DK. Breast cancer patients’ perceptions of their husbands' support in a cross-cultural context. Psycho-Oncol. 2003; 12:24-37. PMid:12548646 http://dx.doi.org/10.1002/pon.619

[18] Avis NE, Crawford S, Manuel J. Psychosocial problems among young women with breast cancer. Psycho-Oncol. 2004; 13:295-308. PMid: 15133771 http://dx.doi.org/10.1002/pon.744

[19] Makabe R, Hull MM. Components of social support among Japanese women with breast cancer. Oncol Nurs Forum. 2000; 27:1381-1390. PMid:11058970

[20] Hashimoto T. Sutoresu to taijinnkankei [Stress and interpersonal relationships]. Kyoto: Nakanishiya Publisher; 2005.

[21] Lazarus, RS, Folkman S. Stress, appraisal, and coping. New York, NY: Springer; 1984.

[22] Makabe R, Nomizu T. Social support and psychological and physical states among Japanese women with breast cancer before and after breast surgery. Oncol Nurs Forum. 2007; 34:883-889. PMid: 17723989 http://dx.doi.org/10.1188/07.ONF.883-889

[23] Makabe R. Nyugan taikennsha no sosharu sapouto to seishinteki • shintaiteki jyoukyou to no kanren [The relationship of social support, psychological, and physical states among Japanese women with breast cancer]. J of Japanese Society of Cancer Nurs. 1998; 12:11-27.

[24] Noguchi Y. Koureisha no sosharu sapoto: sono gainen to sokutei [Social support for elderly: its concept and measurement]. Shakai Ronengaku. 1992; 34:37-48.

[25] Goldberg DP, Hiller VF. A scaled version of the General Health Questionnaire. Psychol Med. 1979; 9:139-145. http://dx. doi.org/10.1017/S0033291700021644

[26] Goldberg D, Williams P. A User's Guide to the General Health Questionnaire: GHQ. Windsor, Berkshire: The NFFR-NELSON Pub Co Ltd. 1988.

[27] Nakagawa T, Daibou I. Seishin kenkou chousa hyou [The Japanese Version of the General Health Questionnaire Manual]. Tokyo: Nihon Bunka Kagakusha. 1985.

[28] Kobayashi E. Koureiki no shakaikannkei [Social relationships in elderly], In T. Gondou. Koureisha Sinnrigaku [Psychology for elderly], Tokyo: Asakura Pub. 2008.

[29] Parrish MM, Satariano WA, Freisthler B, Feinberg LF, Adams S. Older women with breast cancer: caregiving and the risk of depression: an exploratory analysis. Social Work in Health Care. 2005; 40(4):41-59. PMid: 15911503 http://dx.doi.org/10.1300/J010v40n04_03

[30] Stenberg U, Ruland CM, Miaskowski C. Review of the literature on the effects of caring for a patient with cancer.Psycho-Oncol. 2009; 1670p.

[31] Goodwin JS, Satish S, Anderson ET, Nattinger AB, Freeman JL. Effect of nurse case management on the treatment of older women with breast cancer. J of American Geriatric Society. 2003; 51:1252-1259. PMid: 12919237 http://dx.doi.org/10.1046/j.1532- 5415.2003.51409.x 
[32] Von Ah DM, Kang D, Carpenter JS. Predictors of cancer-related fatigue in women with breast cancer before, during, and after adjuvant therapy. Cancer Nurs. 2008; 31:134-144. PMid:18490890 http://dx.doi.org/10.1097/01.NCC.0000305704.84164.54

[33] Makabe R, Ohtake T, Nomizu T. Rounenki nyugan taikensha no sosharu sapoto: Naiyoubunseki niyoru kouseiyouso no kentou [Components of social support among Japanese elderly women with breast cancer: A content analysis of interviews]. Gan kango. 2010; 15:559-565.

[34] Finfgeld-Connett D. Clarification of social support. J of Nurs Scholarship. 2005; 37:4-9. PMid:15813580 http://dx.doi.org/ 10.1111/j.1547-5069.2005.00004.x 